Щербин Сергей Анатольевич, к.т.н., доцент, Ангарский государственный технический университет, e-mail: dekan_ftk@angtu.ru

Глотов Анатолий Андреевич, обучающийся, Ангарский государственный технический университет ОПРЕДЕЛЕНИЕ КАПИТАЛЬНЫХ ЗАТРАТ НА КОЖУХОТРУБЧАТЫЙ ТЕПЛООБМЕННИК

\title{
DETERMINATION OF CAPITAL COSTS FOR SHELL AND TUBE HEAT EXCHANGER
}

Аннотация. Рассмотрены капитальные затраты на кожухотрубчатый теплообменник с учетом его конструктивных параметров и свойств теплоносителей.

Ключевые слова: капитальные затраты, теплообменный аппарат, теплопередача.

Abstract. The capital costs of a shell and tube heat exchanger are considered, taking into account its design parameters and properties of heat carriers.

Keywords: capital costs, heat exchanger, heat transfer.

Кожухотрубчатые теплообменные аппараты (рисунок 1) получили широкое распространение в химической и нефтехимической отраслях промышленности. Это обусловлено их высокой надежностью при работе в широком диапазоне температур и давлений различных технологических сред (жидкостей, газов и паров), относительно простой технологией изготовления и невысокой стоимостью [1].

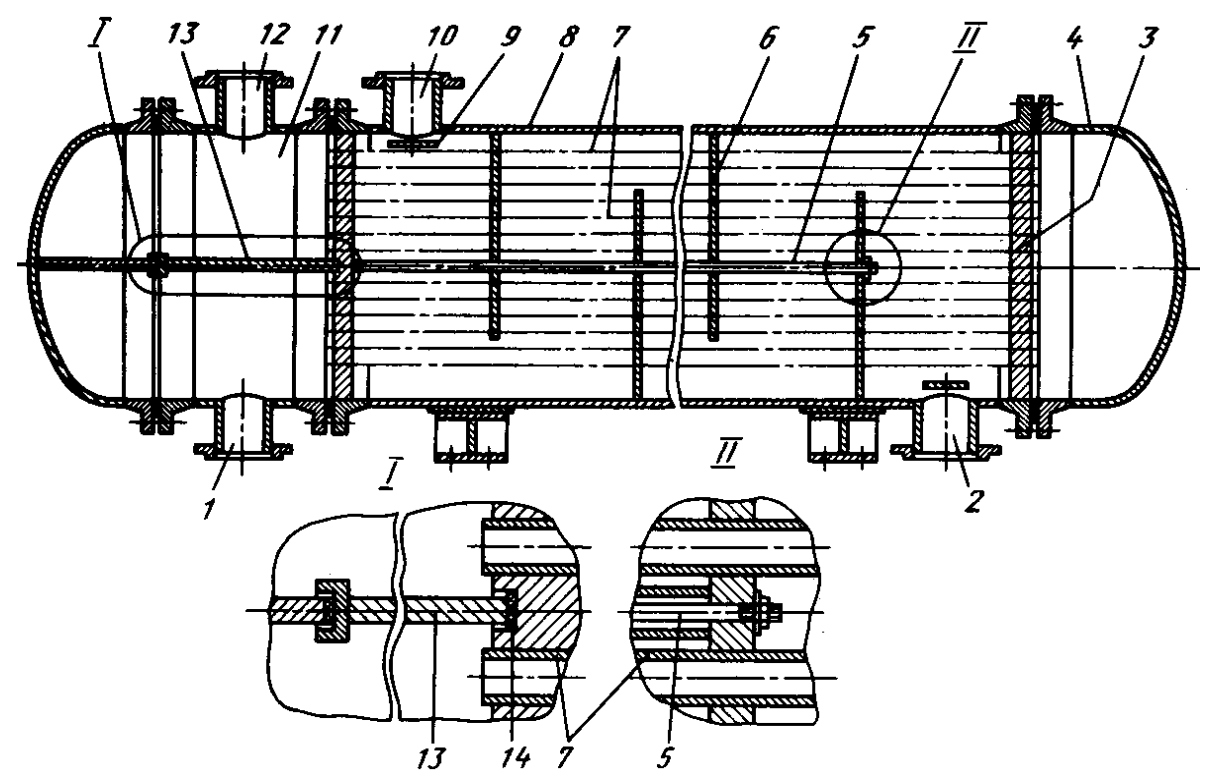

Рисунок 1 - Кожухотрубчатый теплообменник:

1, 12 - штуцера для ввода и вывода теплоносителя из трубного пространства; 2, 10 - штуцера для ввода и вывода теплоносителя из межтрубного пространства; 3 - трубная решетка; 4 - крышка; 5 - стяжка; 6 - сегментная перегородка; 7 - трубы; 8 - кожух; 9 - отбойник; 11 - распределительная камера; 13 - перегородка; 14 - прокладка 
В работе рассматривается подход к определению капитальных затрат на кожухотрубчатый теплообменник с учетом конструктивных характеристик аппарата, а также расходов и свойств теплоносителей.

Капитальные затраты на кожухотрубчатый теплообменник в основном определяются стоимостью труб [2, 3], которая, в свою очередь, зависит от площади поверхности теплообмена $F$, м $^{2}$, обеспечивающей требуемую мощность теплового потока $Q$, Вт. Выразим площадь поверхности теплообмена из основного уравнения теплопередачи:

$$
F=Q /\left(K \cdot \Delta t_{\mathrm{cp}}\right)
$$

где $K$ - коэффрициент теплопередачи, Вт/(м².K):

$$
K=1 /\left(1 / \alpha_{1}+R_{1}+\delta / \lambda_{\text {ст }}+R_{2}+1 / \alpha_{2}\right),
$$

где $\alpha_{1}$ - коэффрициент теплоотдачи от горячего теплоносителя, Вт/(м².К); $R_{1}$ и $R_{2}$ - термические сопротивления загрязнений на поверхностях стенки, $\mathrm{M}^{2} \cdot \mathrm{K} / \mathrm{Bт} ; \delta$ толщина стенки поверхности теплопередачи, м; $\lambda_{\text {ст }}-$ коэффициент теплопроводности материала стенки, Вт/(м.К); $\alpha_{2}$ - коэффицциент теплоотдачи к холод-

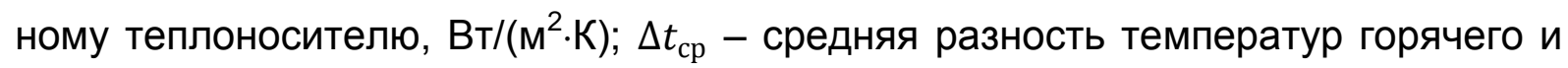
холодного теплоносителей, К.

С учетом количества и геометрических характеристик теплообменных трубок:

$$
F=\pi \cdot\left(d_{1}+d_{2}\right) \cdot l_{\mathrm{T}} \cdot n / 2,
$$

где $d_{1}, d_{2}$ и $l_{\text {т }}$ - соответственно внутренний, наружный диаметры труб аппарата и их длина, м; $n$ - количество теплообменных труб, шт.

Используя (1)-(3), выразим длину труб теплообменника:

$$
l_{\mathrm{T}}=2 \cdot Q /\left[K \cdot \pi \cdot\left(d_{1}+d_{2}\right) \cdot n \cdot \Delta t_{\mathrm{cp}}\right] .
$$

В приведенных далее по тексту выражениях индекс "1" относится к теплоносителю в трубном пространстве аппарата, а индекс "2" - в межтрубном.

При изменении температуры теплоносителя на величину $\Delta t_{1}$, К, мощность теплового потока также можно определить по формуле:

$$
Q=V_{1} \cdot \rho_{1} \cdot c_{1} \cdot \Delta t_{1},
$$

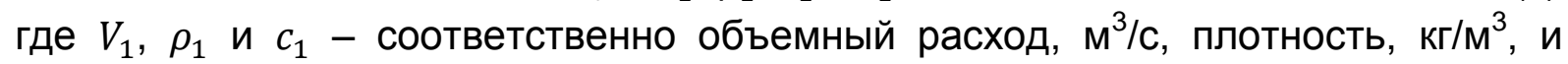
удельная массовая теплоемкость, Дж/(кг·К), теплоносителя.

Объем и масса конструкционного материала теплообменных труб:

$$
\begin{gathered}
V_{\mathrm{T}}=\pi \cdot\left(d_{1}+d_{2}\right) \cdot \delta \cdot l_{\mathrm{T}} / 2 ; \\
m_{\mathrm{T}}=\pi \cdot\left(d_{1}+d_{2}\right) \cdot \delta \cdot l_{\mathrm{T}} \cdot \rho_{\mathrm{M}} / 2,
\end{gathered}
$$

где $\delta$ - толщина стенки трубы, м; $\rho_{\text {м }}$ плотность материала труб, кг/м³.

Единовременные затраты на трубы можно выразить как произведение массы конструкционного материала на стоимость материала труб Ц

Учитывая, что

$$
\mathrm{C}_{\mathrm{T}}=\pi \cdot\left(d_{1}+d_{2}\right) \cdot \delta \cdot l_{\mathrm{T}} \cdot \rho_{\mathrm{M}} \cdot Ц_{\mathrm{T}} / 2 .
$$

$$
\delta=\left(d_{2}-d_{1}\right) / 2,
$$

можно записать: 
и с учетом (4), (5):

$$
\mathrm{C}_{\mathrm{T}}=\pi \cdot\left(d_{2}^{2}-d_{1}^{2}\right) \cdot l_{\mathrm{T}} \cdot \rho_{\mathrm{M}} \cdot Ц_{\mathrm{T}} / 4,
$$

$$
\mathrm{C}_{\mathrm{T}}=\frac{\left(d_{2}-d_{1}\right) \cdot V_{1} \cdot \rho_{1} \cdot \rho_{\mathrm{M}} \cdot c_{1} \cdot \Delta t_{1} \cdot Ц_{\mathrm{T}}}{2 \cdot K \cdot n \cdot \Delta t_{\mathrm{cp}}} .
$$

Коэффрициент $K$ определяется по уравнению (2), в которое входят коэфрфициенты теплоотдачи $\alpha_{1}$ и $\alpha_{2}$, в общем случае определяемые по выражению:

$$
\alpha=N u \cdot \lambda / l \text {, }
$$

где $N u$ - критерий Нуссельта, характеризующий интенсивность теплообмена на границе твердое тело-жидкость; $\lambda$ - коэфффициент теплопроводности теплоносителя, Вт/(м·К); $l$ - определяющий линейный размер поверхности теплообмена, м. В качестве $l$, как правило, принимается длина или диаметр (эквивалентный диаметр) поверхности.

На величину критерия Нуссельта влияет множество фракторов: фризические свойства теплоносителя, скорость и режим его движения, форма, размеры и ориентация поверхности теплообмена в пространстве, температурный напор и другие. Например, при турбулентном режиме движения теплоносителя, агрегатное состояние которого не изменяется, в теплообменных трубках [1]:

$$
N u_{1}=0,021 \cdot R e_{1}^{0,8} \cdot P r_{1}^{0,43},
$$

где $R e_{1}$ - критерий Рейнольдса, характеризующий отношение сил инерции (скоростного напора) к силам вязкого трения и соответственно режим течения теплоносителя; $P r_{1}$ - критерий Прандтля, учитывающий влияние фризических свойств теплоносителя на теплоотдачу:

$$
\begin{gathered}
R e_{1}=W_{1} d_{1} \rho_{1} / \mu_{1}, \\
\operatorname{Pr}_{1}=c_{1} \mu_{1} / \lambda_{1},
\end{gathered}
$$

где $\mu_{1}$ - коэфрфициент динамической вязкости теплоносителя, Па·с; $\lambda_{1}-$ коэфрфрициент теплопроводности теплоносителя, Вт/(м·К); $W_{1}$ - скорость теплоносителя в теплообменных трубах, м/с:

$$
W_{1}=4 \cdot V_{1} \cdot z /\left(n \cdot \pi \cdot d_{1}^{2}\right),
$$

где $z$ - количество ходов по трубам.

Учитывая (12)-(16), после преобразований получим:

$$
\alpha_{1}=\frac{0,025 \cdot V_{1}^{0,8} \cdot z^{0,8} \cdot \rho_{1}^{0,8} \cdot c_{1}^{0,43} \cdot \lambda_{1}^{0,57}}{d_{1}^{1,8} \cdot \mu_{1}^{0,37} \cdot n^{0,8}} .
$$

Критерий Нуссельта при движении теплоносителя в межтрубном пространстве стандартизованного кожухотрубчатого теплообменника с поперечными перегородками при $R e_{2}>10^{3}$ рассчитывается по формуле [1]:

$$
N u_{2}=0,126 \cdot R e_{2}^{0,65} \cdot \operatorname{Pr}_{2}^{0,36} \text {. }
$$

Скорость движения теплоносителя в межтрубном пространстве определяется по его объемному расходу $V_{2}, \mathrm{~m}^{3} / \mathrm{c}$, и площади живого сечения потока:

$$
W_{2}=2 \cdot V_{2} /\left[D^{2} \cdot\left(1-d_{2} / l_{\mathrm{p}}\right)\right] \text {, }
$$

где $D$ - внутренний диаметр кожуха аппарата, $\mathrm{m} ; l_{\mathrm{p}}$ - шаг расположения теплообменных труб в трубной решетке, м. 
Критерий Рейнольдса для теплоносителя в межтрубном пространстве:

$$
R e_{2}=2 \cdot d_{2} \cdot V_{2} \cdot \rho_{2} /\left[D^{2} \cdot\left(1-d_{2} / l_{\mathrm{p}}\right) \cdot \mu_{2}\right] .
$$

Тогда, с учетом (2), (11)-(15), (18) и (20), после преобразований можно записать:

$$
\begin{gathered}
\alpha_{2}=\frac{0,2 \cdot V_{2}^{0,65} \cdot \rho_{2}^{0,65} \cdot c_{2}^{0,36} \cdot \lambda_{2}^{0,64}}{d_{2}^{0,35} \cdot D^{1,3} \cdot \mu_{2}^{0,29}\left(1-d_{2} / l_{\mathrm{p}}\right)^{0,65}} \\
K=\frac{1}{\left(\begin{array}{c}
\frac{d_{1}^{1,8} \cdot \mu_{1}^{0,37} \cdot n^{0,8}}{0,025 \cdot V_{1}^{0,8} \cdot z^{0,8} \cdot \rho_{1}^{0,8} \cdot c_{1}^{0,43} \cdot \lambda_{1}^{0,57}}+R_{1}+\frac{d_{2}-d_{1}}{2 \cdot \lambda_{\mathrm{cT}}}+R_{2}+ \\
+\frac{d_{2}^{0,35} \cdot D^{1,3} \cdot \mu_{2}^{0,29}\left(1-d_{2} / l_{\mathrm{p}}\right)^{0,65}}{0,2 \cdot V_{2}^{0,65} \cdot \rho_{2}^{0,65} \cdot c_{2}^{0,36} \cdot \lambda_{2}^{0,64}}
\end{array}\right)} \\
\cdot\left(\begin{array}{c}
\frac{\left(d_{2}-d_{1}\right) \cdot V_{1} \cdot \rho_{1} \cdot \rho_{\mathrm{M}} \cdot c_{1} \cdot \Delta t_{1} \cdot \bigsqcup_{\mathrm{T}}}{2 \cdot n \cdot \Delta t_{\mathrm{cp}}} \\
d_{1}^{1,8} \cdot \mu_{1}^{0,37} \\
0,025 \cdot c_{1}^{0,43} \cdot \lambda_{1}^{0,57} \cdot\left(\frac{n}{V_{1} \cdot \rho_{1} \cdot z}\right)^{0,8}+R_{1}+\frac{d_{2}-d_{1}}{2 \cdot \lambda_{\mathrm{cr}}}+R_{2}+ \\
+\frac{d_{2}^{0,35} \cdot D^{1,3} \cdot \mu_{2}^{0,29}}{0,2 \cdot c_{2}^{0,36} \cdot \lambda_{2}^{0,64}} \cdot\left(\frac{\left(1-d_{2} / l_{\mathrm{p}}\right)}{V_{2} \cdot \rho_{2}}\right)^{0,65}
\end{array}\right)
\end{gathered}
$$

Полученное выражение (23) позволяет оценить капитальные затраты на кожухотрубчатый теплообменник с учетом расходов и свойств теплоносителей, а также конструктивных характеристик аппарата. На следующем этапе работы предстоит апробация предложенного подхода на стадии проектирования теплообменных аппаратов с целью проверки его эффективности.

\section{ЛИТЕРАТУРА}

1. Щербин С.А. Основы теории теплообмена и теплообменные аппараты. - Учебное пособие. Ангарск: АГТА, 2014. 162 с.

2. Кузьмин С.И., Щербин С.А. Комплексная оптимизация трубчатого теплообменника // Сборник научных трудов АнГТУ. 2018. С. 21-27.

3. Щербин С.А., Демин Г.Г. Вопросы оптимизации теплообменных аппаратов // Вестник АнГТУ. 2019. № 13. С. 106-109. 